\title{
Unstable drift-kinetic Alfvén modes with helicity close to the rotational transform
}

\author{
D.Yu. Eremin \\ Max-Plank-Institut für Plasmaphysik, \\ EUROATOM-Association, D-17491 Greifswald, Germany*
}

\begin{abstract}
An important feature of some modern facilities (such as optimized stellarators) with low shear is that the value of their rotational transform is close to a rational number. If a mode helicity is close to the rotational transform of the background magnetic field, the Alfvén continuum frequency can lie in the range of the diamagnetic frequencies of the background electrons. An analytical analysis shows that if the local electron diamagnetic frequency curve crosses the Alfvén continuum from above as one proceeds from the axis to the plasma edge at a radial location relatively distant from the axis, a family of unstable drift-kinetic Aflvén modes (DKAEs) arises due to the coupling between the drift and Alfvén waves having same poloidal numbers. The coupling is mediated by the parallel electric field and the mode is destabilized due to parallel resonances. The mode growth rate can be relatively large and comparable to the real part of the mode frequency. The growth rate peaks at a small radial number, so that the most unstable mode can appear global and be confused with the magnetohydrodynamic (MHD) modes which can occur in this part of the spectrum. It follows that it is important to consider the diamagnetic effects and finite parallel electric field, which are frequently neglected for such phenomena in tokamaks, when studying Alfvén phenomena in the lowest part of the electromagnetic spectrum in optimized stellarators.
\end{abstract}

PACS numbers: $52.35 .-\mathrm{g}, 52.30 . \mathrm{Gz}, 52.65 . \mathrm{Tt}, 52.55 . \mathrm{Pi}$

*Electronic address: dee@ipp.mpg.de 


\section{INTRODUCTION}

A large number of the experimental data in partially and fully optimized stellarator facilities indicates unstable modes in the lowest part of the Alfvénic spectrum (e.g., Refs. [1],[2],[3]). It is therefore important to understand which varieties of electromagnetic modes can in principle exist there and whether they can become unstable.

A salient feature of a number of existing or planned modern stellarator facilities is the low shear of the rotational transform of the magnetic field to avoid low order resonant rational surfaces and thus suppress formation of magnetic islands and undesirable magnetohydrodynamic (MHD) activity. In such facilities the value of the rotational transform is close to a low order rational number in the entire radial domain. It follows that the shear Alfvén continuum of a mode with helicity close to the rotational transform can lie in the range of very small frequencies, comparable to the local diamagnetic frequencies of the background species. One may assume then that shear Alfvén waves and drift waves can strongly interact with each other through the parallel electric field at the locations where their local frequencies match, giving rise to drift-kinetic Alfvén modes (DKAEs) ([4]). Since the sign of the energy transfer in the parallel Landau resonant wave-particle interaction is determined by the sign of the factor $\omega_{*} / \omega-1$, where $\omega_{*}$ is diamagnetic frequency of a background species, such a mode can become unstable when the condition $\omega_{*}>\omega$ holds in a significant part of a plasma. Therefore, such a mode is, generally speaking, non-perturbative, because its existence and growth are brought about by the same kinetic effects connected to the parallel electric field.

In this paper we perform a local analysis of the DKAE with helicity close to the rotational transform in an inhomogeneous plasma of an optimized stellarator. The DKAE considered here is produced by coupling between the shear Alfvén wave and the electron drift wave having the same poloidal numbers determined by the closeness of the mode helicities to the rotational transform. The paper is organized as follows: in Sec. II we derive equations governing the shear-Alfvén dynamics with the parallel electric field taken into account, which enables us to describe the coupling between shear-Alfvén and drift modes with the same mode numbers and to justify the neglect of the sideband response for the modes in question, in Sec. III we study the equations of Sec. II in the vicinity of a point where the shear Alfvén continuum and the local electron diamagnetic drift frequencies match, we demonstrate that 
there exist unstable DKAEs with the real part of the frequency lying above the minimum of the shear Alfvén continuum and the growth rate comparable to the real frequency, in Sec. IV we verify predictions of the local analysis of Sec. III with numerical solution of the general equations of Sec. II for a case with parameters relevant to the Helically Symmetric Experiment (HSX, see Ref. [5]), and in Sec. V we draw conclusions.

\section{SHEAR ALFVÉN DYNAMICS WITH FINITE PARALLEL ELECTRIC FIELD}

To derive the equations governing the low-frequency shear Alfvén dynamics in a toroidal plasma with finite $E_{\|}$taken into account, we start from the the ansatz of a plasma with low beta $\left(\beta \simeq m_{e} / m_{i}\right.$, where $\beta=2 \mu_{0} p / B^{2}$ is the ratio between the kinetic and magnetic pressures), hot electrons and cold ions $\left(T_{e} \gg T_{i}\right)$. We consider a background plasma with nonuniform density but constant temperature. The analysis conducted in this paper can easily be generalized to the case when both density and temperature have radial profiles. The distribution function of the background plasma is assumed to be Maxwellian. For the mode of interest the mode numbers satisfy $m \iota-n \ll 1$.

We employ magnetic Boozer coordinates $(\psi, \theta, \phi)$ (Ref. [6]) to represent the magnetic field, neglecting $B_{\psi}$, which is related to Pfirsch-Schlüter current and generated by the pressure effects $\left(B_{\psi} / B_{\phi}=\mathcal{O}(\beta)[7]\right.$, where $B_{\phi} \equiv \bar{B} R_{0}=$ const $)$. Further, similarly to Ref. [8], one can assume that $B_{\theta} / B_{\phi}=\mathcal{O}\left(a^{2} / R_{0}^{2}\right)$, where $a / R_{0}$ is the "average" aspect ratio, $B^{2} J \approx B_{\phi}$ with $1 / J=[\nabla \psi \times \nabla \theta] \cdot \nabla \phi$. Note that in an ideal current-less stellarator $B_{\theta}$, which basically represents the toroidal current, would have been zero. Thus, in the covariant representation the background magnetic field is $\mathbf{B} \approx B_{\phi} \nabla \phi$. Analogously to Ref. [9] we assume the magnetic field strength to be $B=\bar{B} h_{B}, h_{B}=1+\frac{1}{2} \sum_{\mu, \nu} \epsilon_{B}^{\mu, \nu} e^{i(\mu \theta-\nu N \phi)}$ with $\epsilon_{B}^{\mu, \nu}=\epsilon_{B}^{-\mu,-\nu} \ll 1$ and $N$ the number of field periods, and dominant components of the contravariant metric to be $g^{\psi \psi}=2 \delta \bar{B} \psi h_{g}^{\psi \psi}$ and $g^{\theta \theta}=\frac{\delta \bar{B}}{2 \psi} h_{g}^{\theta \theta}$, and $g^{\psi \theta}=\delta \bar{B} h_{g}^{\psi \theta}$ with $h_{g}^{i j}=\delta^{i j}+\frac{1}{2} \sum_{\mu, \nu} \epsilon_{g}^{i j(\mu, \nu)} e^{i(\mu \theta-\nu N \phi)}$, $\delta^{i j}$ the Kronecker symbol, $\delta=\left(\kappa+\kappa^{-1}\right) / 2$ with $\kappa$ the elongation, $\epsilon_{g}^{i j(\mu, \nu)}=\epsilon_{g}^{i j(-\mu,-\nu)}$, and the other components being smaller by factor of order $\mathcal{O}\left(a^{2} / R_{0}^{2}\right)$ (Ref. [8]).

As a first step, this paper considers only interaction of the drift-kinetic Alfvén modes with well-circulating particles, the trapped particle contribution being the subject of a separate study, and omits compressional effects.

It follows that the parallel component of the perturbed magnetic field can be set to 
zero, and the perpendicular one can be described in terms of the parallel component of the vector potential, so that the magnetic field perturbation is $\tilde{\mathbf{B}}=\left[\nabla A_{\|} \times \mathbf{b}\right]$ with $\mathbf{b}=\mathbf{B} / B$. By linearizing the gyrokinetic equation in the limit of zero Larmor radius (although the FLR effects are insignificant for this problem, they can in principle be trivially recovered by applying the gyrokinetic averaging to the corresponding potentials) for both background species in the presence of a low-frequency electromagnetic wave (e.g., [10]), one obtains

$$
\frac{\partial \delta f_{s}}{\partial t}+\frac{d^{(0)} \mathbf{R}}{d t} \frac{\partial \delta f_{s}}{\partial \mathbf{R}}+\frac{d^{(0)} v_{\|}}{d t} \frac{\partial \delta f_{s}}{\partial v_{\|}}=-\frac{d^{(1)} \mathbf{R}}{d t} \frac{\partial f_{0 s}}{\partial \mathbf{R}}-\frac{d^{(1)} v_{\|}}{d t} \frac{\partial f_{0 s}}{\partial v_{\|}},
$$

where $\delta f_{s}=f_{s}-f_{0 s}$, with $s=i, e$, is the deviation of the distribution function from Maxwellian $f_{0 s}, \mu=m_{s} v_{\perp}^{2} / 2 B$, index (0) indicates that the derivative is taken along the unperturbed motion,

$$
\begin{aligned}
& \frac{d^{(0)} \mathbf{R}}{d t}=v_{\|}\left(\mathbf{b}+\frac{m_{s} v_{\|}}{q_{s} B}[\nabla \times \mathbf{b}]\right)+\frac{[\mathbf{b} \times \mu \nabla B]}{q_{s} B} \\
& \frac{d^{(0)} v_{\|}}{d t}=-\left(\mathbf{b}+\frac{m_{s} v_{\|}}{q_{s} B}[\nabla \times \mathbf{b}]\right) \cdot \frac{\mu \nabla B}{m_{s}},
\end{aligned}
$$

with $\Phi$ the electrostatic potential, and index (1) points out that the derivation is made along the perturbations of the particle orbits in phase space due to the electromagnetic field,

$$
\begin{aligned}
\frac{d^{(1)} \mathbf{R}}{d t} & =v_{\|} \frac{\left[\nabla A_{\|} \times \mathbf{b}\right]}{B}-\frac{[\nabla \Phi \times \mathbf{b}]}{B} \\
\frac{d^{(1)} v_{\|}}{d t} & =-\frac{\left[\nabla A_{\|} \times \mathbf{b}\right] \cdot \mu \nabla B}{m_{s} B}-\frac{q_{s}}{m_{s}}\left(\left(\mathbf{b}+\frac{m_{s} v_{\|}}{q_{s} B}[\nabla \times \mathbf{b}]\right) \cdot \nabla \Phi+\frac{\partial A_{\|}}{\partial t}\right),
\end{aligned}
$$

and in derivation of these equations we used $\mathbf{b} \cdot[\nabla \times \mathbf{b}] \approx-\frac{B_{\phi}^{2}}{B^{2}} \nabla \phi \cdot[\nabla B \times \nabla \phi]=0$.

To make the description self-consistent one needs to describe influence of the plasma on the electromagnetic fields. To this end, it is appropriate to use the quasineutrality equation,

$$
-\nabla \cdot\left(\frac{n m_{i}}{B^{2}} \nabla_{\perp} \Phi\right)=\sum_{s} q_{s} \delta n_{s}
$$

where $\delta n_{s}=\int \delta f_{s} d^{3} \mathbf{v}$ with $d^{3} \mathbf{v}=2 \pi \frac{B}{m_{s}} d v_{\|} d \mu$, and the parallel Ampere's law,

$$
\mathbf{b} \cdot[\nabla \times \tilde{\mathbf{B}}]=\mu_{0} \sum_{s} \delta j_{\| s}
$$

where $\delta j_{\| s}=q_{s} \int v_{\|} \delta f_{s} d^{3} \mathbf{v}$. As is demonstrated in Ref. [8], the left hand side of Eq. (5) equals to $-\frac{1}{B} \nabla \cdot B^{2} \nabla_{\perp} \frac{A_{\|}}{B}$ in case of small background pressure.

We are looking for the normal modes of the system,

$$
\left(\Phi, A_{\|}\right)=\sum_{m, n}\left(\Phi^{m, n}(\psi), A_{\|}^{m, n}(\psi)\right) e^{i(-\omega t+m \theta-n \phi)},
$$


with $\psi$ the flux label. To obtain the eigenfunction equations governing the coefficients $\Phi_{m n}$ and $A_{\| m n}$, we first integrate Eq. (1) multiplied by $q_{s}$ over $v_{\|}$and $\mu$, and then add the resulting equations using Eqs. (4) and (5). This procedure leads to

$$
\begin{aligned}
\omega^{2} \nabla \cdot\left(\frac{1}{v_{A}^{2}} \nabla_{\perp} \Phi\right) & +\nabla \cdot\left[\frac{\mathbf{b}}{B} \nabla \cdot\left(B^{2} \nabla_{\perp} \frac{\nabla_{\|} \Phi}{B}\right)\right]-\mu_{0}\left[\nabla \nabla_{\|} \Phi \times \mathbf{b}\right] \cdot \nabla_{\frac{j_{0}}{B}} \\
& =i \omega \mu_{0} \sum_{s} q_{s} \int\left(\mathbf{v}_{\mathbf{d s}} \cdot \nabla\right) \delta f_{s} d^{3} \mathbf{v}+\nabla \cdot\left[\frac{\mathbf{b}}{B} \nabla \cdot\left(B^{2} \nabla_{\perp} \frac{\nabla_{\|}(\Phi-\Psi)}{B}\right)\right]
\end{aligned}
$$

with $v_{A}^{2}=B_{0}^{2} / \mu_{0} n m_{i}, \mathbf{v}_{\mathbf{d s}}=\frac{\left(v_{\|}^{2}+v_{\perp}^{2} / 2\right)}{\Omega_{s}} \frac{[\mathbf{b} \times \nabla B]}{B}$, and $A_{\|}$is expressed as $A_{\|}=\frac{1}{i \omega} \nabla_{\|} \Psi$, which is basically the well-known vorticity equation in ideal MHD in the appropriate limit of low beta but for the term arising from particle drifts and the term proportional to the parallel electric field on the right hand side. In this equation we kept only one term which depends on $\nabla_{\|}(\Phi-\Psi)$ and consequently describes the coupling with the parallel electric field. As we will see later, if one expresses this term in terms of $\Phi$ using an equation for the parallel electric field which we will derive shortly, it will result in a fourth-order radial derivative of $\Phi$ and thus will allow for the existence of discrete modes. In the same way, the other term depending on $\nabla_{\|}(\Phi-\Psi)$ does not vanish only provided the parallel background plasma current is not zero, leads to the second radial derivative of $\Phi$, and thus does not significantly alter the resulting modes. Henceforth we drop this term.

By using $b^{\phi}=1 / J B, b^{\theta}=\iota(\psi) / J B$, and $\nabla_{\|}=h_{B} \hat{L} / R_{0}$ with $\hat{L}=\partial / \partial \phi+\iota \partial / \partial \theta$, Eq. (7) in the coordinate representation becomes

$$
\begin{aligned}
& \hat{L} \frac{\partial}{\partial x^{i}}\left(g^{i j} \frac{\partial}{\partial x^{j}}\left(k_{\|} \Phi\right)-i b^{i} k_{\|}^{2} h_{B} \Phi\right)-\omega^{2} R_{0} \frac{\partial}{\partial x^{i}} \frac{1}{v_{A}^{2} h_{B}^{4}}\left(i g^{i j} \frac{\partial}{\partial x^{i}}+b^{i} k_{\|} h_{B}\right) \Phi-\mu_{0} \frac{k_{\|} R_{0}}{h_{B}} \frac{\partial}{\partial \theta}\left(h_{B} \Phi\right) \frac{d j_{0 \|} / h_{B}}{d \psi} \\
& =\frac{\mu_{0} \omega R_{0}}{h_{B}} \sum_{s} q_{s} \int\left(\mathbf{v}_{\mathbf{d s}} \cdot \nabla\right) \delta f_{s} d^{3} \mathbf{v}+\hat{L} \frac{\partial}{\partial x^{i}}\left(g^{i j} \frac{\partial}{\partial x^{j}}\left(k_{\|}(\Phi-\Psi)\right)-i b^{i} k_{\|}^{2} h_{B}(\Phi-\Psi)\right)
\end{aligned}
$$

where $k_{\|}(\psi)=(m \iota(\psi)-n) / R_{0}, \overline{v_{A}^{2}}(\psi)=\bar{B} / \mu_{0} n(\psi) m_{i}$, and in the coordinate representation $\mathbf{v}_{\mathbf{d s}}=\frac{m_{s}}{q_{s} h_{B}}\left(\mathbf{e}_{\theta} \frac{\partial h_{B}}{\partial \psi}-\mathbf{e}_{\psi} \frac{\partial h_{B}}{\partial \theta}\right)$. To simplify this equation we note that the terms of order $\mathcal{O}(\epsilon)$ (with $\epsilon=\max \left(a / R_{0}, \epsilon_{B}, \epsilon_{g}\right)$ ) lead to the coupling of a mode $(m, n)$ to the modes with different poloidal and toroidal numbers, $(m+\mu, n+\nu N)$. However, the mode we are interested in with the mode numbers $(m, n)$ satisfying $n / m \approx \iota$ has frequency much lower than the Alfvén continuum frequencies for the modes with other mode numbers. Besides that, we assume that the mode is local enough not to be affected by the other drift modes. Consequently, we neglect the coupling to the modes with different mode numbers in Eq. (8) and take $h_{B}$ along with $h_{g}^{i j}$ to be approximately unity everywhere in this equation but the 
expression for $\mathbf{v}_{\mathbf{d s}}$ where we keep the full form for $h_{B}$ because there remains a possibility that the mode is affected by the self-driven sideband response through the first term on the right hand of Eq. (8). To compare this contribution with the contribution from the parallel electric field given by the second term of the right hand side of Eq. (8), we re-write Eq. (1) similarly to Refs. [11] and [12] as

$$
\left(-i \omega+v_{\|} \nabla_{\|}+\left(\mathbf{v}_{\mathbf{d s}} \cdot \nabla\right)\right) g_{s}=\frac{q_{s} f_{0 s}}{T_{s}}\left(1-\frac{\hat{\omega}_{* s}}{\omega}\right)\left(v_{\|} E_{\|}+\mathbf{v}_{\mathbf{d s}} \cdot \mathbf{E}_{\perp}\right),
$$

where $E_{\|}=-\nabla_{\|} \Phi-\frac{\partial A_{\|}}{\partial t}, \mathbf{E}_{\perp}=-\nabla_{\perp} \Phi$, and $g_{s}=\delta f_{s}-\frac{[\nabla \Phi \times \mathbf{b}] \cdot \nabla f_{0 s}}{i \omega B}$ with the second term in this expression representing the "sloshing" non-resonant part of the perturbed distribution function, which does not contribute to the resonant wave-particle interaction and thus to the mode growth. Hence, the corresponding contribution to the first term in Eq. (8) is of no significance. Additionally, $\hat{\omega}_{* s} \equiv \frac{i T_{s}}{q_{s} \bar{B} r} \chi_{n} \frac{\partial}{\partial \theta}$, where we introduced a new radial coordinate $r=(2 \psi / \bar{B})^{1 / 2}, \chi_{n}=-(d n / d r) / n$, and this operator acts only on the potentials $\Phi$ and $\Psi$. In derivation of Eq. (9) we assumed $\left(d^{2} n / d r^{2}\right) /(d n / d r) \ll \Phi /(d \Phi / d r)$. Then, proceeding in the spirit of Ref. [12], one can write equations for the coupling of the $(m, n)$ mode with the $(m+\mu, n+\nu N)$ sideband response (here each of $\mu$ and $\nu$ can be either positive or negative) arising due to a $(\mu, \nu)$ harmonic in the spectrum of the magnetic field strength in Boozer coordinates,

$$
\begin{aligned}
(-\omega+ & \left.k_{\|}^{m, n} v_{\|}\right) g_{s}^{m, n}+v_{d s} \hat{k}_{\perp}^{m+\mu, \mu} g_{s}^{m+\mu, n+\nu N} \\
& =-\frac{q_{s}}{T_{s}} f_{0 s} v_{\|}\left(1-\frac{\bar{\omega}_{* s}^{m}}{\omega}\right) k_{\|}^{m, n}\left(\Phi^{m, n}-\Psi^{m, n}\right)-\frac{q_{s}}{T_{s}} f_{0 s} v_{d s}\left(1-\frac{\bar{\omega}_{* s}^{m+\mu}}{\omega}\right) \hat{k}_{\perp}^{m+\mu, \mu} \Phi^{m+\mu, n+\nu N}
\end{aligned}
$$

and

$$
\begin{aligned}
(-\omega+ & \left.k_{\|}^{m+\mu, n+\nu N} v_{\|}\right) g_{s}^{m+\mu, n+\nu N}+v_{d s} \hat{k}_{\perp}^{m,-\mu} g_{s}^{m, n} \\
= & -\frac{q_{s}}{T_{s}} f_{0 s} v_{\|}\left(1-\frac{\bar{\omega}_{* s}^{m+\mu}}{\omega}\right) k_{\|}^{m+\mu, n+\nu N}\left(\Phi^{m+\mu, n+\nu N}-\Psi^{m+\mu, n+\nu N}\right) \\
& \quad-\frac{q_{s}}{T_{s}} f_{0 s} v_{d s}\left(1-\frac{\bar{\omega}_{* s}^{m}}{\omega}\right) \hat{k}_{\perp}^{m,-\mu} \Phi^{m, n},
\end{aligned}
$$

where for each term we took just first non-vanishing terms in terms of $\epsilon, \bar{\omega}_{* s}^{m}=-\frac{m T_{s}}{q_{s} \bar{B} r} \chi_{n}$, $v_{d s}=\frac{m_{s}}{q_{s} \bar{B} R_{0}}\left(v_{\|}^{2}+v_{\perp}^{2} / 2\right), k_{\|}^{m, n}=(m \iota-n) / R_{0}$, and $\hat{k}_{\perp}^{m, n}=\frac{R_{0}}{2 r}\left(m \frac{\partial \epsilon_{B}^{\mu, \nu}}{\partial r}+n \epsilon_{B}^{\mu, \nu} \frac{\partial}{\partial r}\right)$. Under the assumption of cold ions and after the neglect of the coupling with the $(m+\mu, n+\nu N)$ components of the potentials as has been argued above, there only remains $(m+\mu, n+$ $\nu N)$ sideband response of the perturbed electron distribution function driven by the $(m, n)$ component of the potentials. This generates the $(m, n)$ perturbed current response through the $(-\mu,-\nu N)$ harmonic of the $\mathbf{v}_{\mathbf{d e}}$ in the first term on the right hand side of Eq. (8). 
Similarly to Ref. [12], by using the ordering parameter $\left(\mathbf{k}_{\perp} \cdot \mathbf{v}_{\mathbf{d e}}\right) / \omega \ll 1$, one can neglect the second terms on the left hand side of Eqs. (10) and (11), after which it is straightforward to solve for the $g_{e}^{m, n}$ and $g_{e}^{m+\mu, n+\nu N}$ in terms of $\Phi^{m, n}$ and $\Psi^{m, n}$. Then, by substituting the resulting expressions into the first and second terms on the right hand side of Eq. (11) (in the course of this procedure $\left(\Phi^{m, n}-\Psi^{m, n}\right)$ is to be determined from the corresponding quasineutrality equation Eq. (4), see also Eq. (14) ), the ratio of the imaginary contribution from the first term to that of the second term can be estimated as $\mathcal{O}\left(\left(1-\frac{\bar{\omega}_{* e}^{m}}{\omega}\right)^{2} \frac{1}{k_{r}^{2} \rho_{s}^{2}} \frac{m_{e}}{m_{i}}\right) \ll$ 1 , where, as we will see below in Sec. III, $k_{r}$ can be estimated as $k_{r}=d / d r \approx 1 / a \sigma^{1 / 4} \gg 1 / a$ where $\sigma$ is defined after Eq. (16). In deriving this estimate the asymptotic of $Z$ function for small arguments, $k_{\|}^{m, n} V_{A} \approx \omega, k^{m+\mu, n+\nu N} R_{0}=\mathcal{O}(1)$, and $v_{\text {the }} \approx V_{A}$ were used. Note also that for the DKAEs $\omega \approx \bar{\omega}_{* e}^{m}$. Therefore, one can conclude that the sideband coupling in Eqs. (1) and (7) can be neglected altogether by imposing $h_{B}=1$ and $h_{g}^{i j}=\delta^{i j}$ and correspondingly $v_{d e}=0$ everywhere in these equations. Since no coupling will be considered, from this point on we will write these equations for the $(m, n)$ harmonic, suppressing the $(m, n)$ subscripts in the notations.

We proceed with separation of the rotational transform generated by the external magnetic coils $\iota_{\text {ext }}$ as suggested in Ref. [17], repeating briefly the arguments given in the reference. By writing the rotational transform as $\iota=\frac{B^{\theta}}{B^{\Phi}}=\frac{g^{\theta \theta}}{B^{\Phi}}+\frac{g^{\theta \Phi}}{B^{\theta}} \equiv \iota_{e x t}+\frac{g^{\theta \Phi}}{B^{\theta}} \equiv \iota_{e x t}+\nu_{\iota} \iota$, one can separate the part of the rotational transform generated by the external coils (denoted as $\iota_{\text {ext }}$ ), and write the part of the rotational transform produced by the plasma current as $\nu_{\iota} \iota$. Expressing further the parallel background plasma current as $j_{0 \|}=\frac{\bar{B}}{\mu_{0} R_{0} r} \frac{d}{d r} r^{2} \nu_{\iota} \iota$, Eq. (8) yields

$$
\begin{aligned}
\frac{1}{r^{2}} \frac{\partial}{\partial r} r^{3}\left(\frac{\omega^{2}}{\overline{v_{A}^{2}}}-k_{\|}^{2}\right) \frac{\partial}{\partial r} \frac{\Phi}{r} & -\frac{\left(m^{2}-1\right)}{r^{2}}\left(\frac{\omega^{2}}{\overline{v_{A}^{2}}}-k_{\|}^{2}\right) \Phi+G(r) \Phi \\
& =\left(-\frac{k_{\|} m^{2}}{r^{2}}+\frac{k_{\|}}{r} \frac{\partial}{\partial r} r \frac{\partial}{\partial r}\right) k_{\|}(\Psi-\Phi)
\end{aligned}
$$

where $G(r)=\frac{\omega^{2}}{r}\left(\frac{1}{v_{A}^{2}}\right)^{\prime}+\frac{m k_{\|}}{r R_{0}}\left(\left(3 \iota^{\prime}+r \iota^{\prime \prime}\right)\left(\nu_{\iota}-1\right)+\left(2 r \iota^{\prime}+3 \iota\right) \nu_{\iota}^{\prime}+r \iota \nu_{\iota}^{\prime \prime}\right)$. When $\nu_{\iota}=1$, i.e. the entire rotational transform is generated by the plasma current, and $\Phi=\Psi$, Eq. (12) agrees with the well-known MHD counterpart for tokamaks (e.g., Eq. (33) in Ref. [12]). The case with $\nu_{\iota}=0$ corresponds to a currentless stellarator.

To make the system self-consistent, one has also to obtain an equation for the parallel electric field. To this end we solve the Eq. (1), which yields

$$
\delta f_{s}=-\frac{q_{s}}{T_{s}} f_{0 s}\left[\frac{\omega_{* s}}{\omega} \Psi+\left(1+\frac{\omega-\omega_{* s}}{k_{\|} v_{\|}-\omega}\right)(\Phi-\Psi)\right],
$$


where $\omega_{* s}=\bar{\omega}_{* s}^{m}$. Further, we insert the distribution function perturbation from Eq. (13) into the quasineutrality equation (Eq. (4)), which results in

$$
(\Phi-\Psi)\left(1-\frac{\omega_{* e}}{\omega}\right) \frac{\left(1+\zeta_{e} Z\left(\zeta_{e}\right)\right)}{\overline{v_{A}^{2}}}=\rho_{s}^{2}\left(-\frac{m^{2}}{r^{2} \overline{v_{A}^{2}}}+\frac{1}{r} \frac{\partial}{\partial r} \frac{r}{\overline{v_{A}^{2}}} \frac{\partial}{\partial r}\right) \Phi
$$

where $\rho_{s}=c_{s} / \Omega_{i}, c_{s}=\sqrt{T_{e} / m_{i}}, \Omega_{i}=e \bar{B} / m_{i}, \zeta_{e}=\omega m_{e}^{1 / 2} / k_{\|}\left(2 T_{e}\right)^{1 / 2}$, and $Z(\zeta)=$ $\frac{1}{\sqrt{\pi}} \int_{-\infty}^{\infty} d t e^{-t^{2}} /(t-\zeta)$ is the plasma dispersion function (e.g., Ref. [15]). In deriving this equation we assumed that the background ions are cold, i.e. $\sqrt{T_{i} / m_{i}} \ll v_{A}$ and that $k_{\|} c_{s} \ll \omega$, so that one can neglect the ion contribution in the parallel plasma response on the left hand side. Eqs. (12) and (14) describe normal modes in the system.

\section{DRIFT-KINETIC ALFVÉN MODES}

To see how the coupling between a shear Alfvén wave and a drift wave gives rise to a family of discrete drift-Alfvén modes we first note that such a mode should spatially reside close to the radial position $r_{0}$ where the local electron diamagnetic frequency is equal to the local frequency of the Alfvén continuum, i.e. $\omega_{0} \equiv \omega_{* e}\left(r_{0}\right)=k_{\|}\left(r_{0}\right) \overline{v_{A}}\left(r_{0}\right)$. The mode frequency should also be close to $\omega_{0}$. Consequently, we expand the functions $F \equiv \omega^{2} / \overline{v_{A}^{2}}(r)-k_{\|}^{2}(r)$ in Eq. (12) and $\omega-\omega_{* e}(r)$ in Eq. (14) in Taylor series around $r_{0}$ in the radial domain and around $\omega_{0}$ in the frequency domain, and take all other quantities at $r_{0}$ constant with the exception of $G(r)$, which we also expand up to the first order. The latter term is important in the treatment of the modes with $m=1$, as will become clear later.

Following this procedure, one obtains

$$
\left\{\begin{array}{c}
(x-\delta)(\Psi-\Phi)=\frac{1}{\left(1+\zeta_{e} Z\left(\zeta_{e}\right)\right)} \frac{\omega}{\omega_{* e}^{\prime} a} \frac{\rho_{s}^{2}}{a^{2}}\left(\frac{d^{2}}{d x^{2}}-k_{\perp}^{2} a^{2}\right) \Phi \\
\frac{d}{d x} x \frac{d}{d x} \Phi-f x \Phi+g \Phi=\frac{k_{\|}^{2}}{F_{0}^{\prime} a}\left(\frac{d^{2}}{d x^{2}}-k_{\perp}^{2} a^{2}\right)(\Psi-\Phi)
\end{array}\right.
$$

here $x=\frac{r-r_{0}}{a}+\frac{F_{0}}{F_{0}^{\prime} a}, k_{\perp}=\frac{m}{r_{0}}, F_{0}=\frac{\left(\omega^{2}-\omega_{0}^{2}\right)}{\overline{v_{A 0}^{2}}}, F_{0}^{\prime}=\left(\frac{1}{\bar{v}_{A}^{2}}\right)_{0}^{\prime}\left(\omega^{2}-\omega_{0}^{2}\right)-\frac{\left(k_{\|}^{2} \overline{v_{A}^{2}}\right)_{0}^{\prime}}{\overline{v_{A 0}^{2}}}, \delta=\frac{F_{0}}{F_{0}^{\prime} a}+\frac{\left(\omega-\omega_{0}\right)}{\omega_{* e 0}^{\prime} a}$, prime denotes the radial derivative and all quantities with the subscript " 0 " are taken at $r=r_{0}, f$ and $g$ are defined in Appendix A. The following analysis assumes that $-\infty<x<$ $\infty$, which can hold only if $\omega^{2}>\left(k_{\|}^{2} \overline{v_{A}^{2}}\right)_{\text {min. }}$. Eq. (15) must then be solved subject to the boundary conditions $\Phi(x \rightarrow \pm \infty)=\Psi(x \rightarrow \pm \infty)=0$.

Further, we apply a Fourier transform defined by $\hat{\Phi}=\int_{-\infty}^{\infty} d x e^{-i k x} \Phi$ to Eq. (15). After 
combining the resulting equations into one, we obtain

$$
\begin{aligned}
\frac{d^{2} \hat{\Phi}}{d k^{2}} & +\frac{d \hat{\Phi}}{d k}\left(\frac{k^{3}+k\left(3 k_{\perp}^{2} a^{2}-2 f\right)}{\left(k^{2}+f\right)\left(k^{2}+k_{\perp}^{2} a^{2}\right)}+i \delta+\frac{i g}{k^{2}+f}\right) \\
& +\hat{\Phi}\left(\frac{k_{\perp}^{2} a^{2}-k^{2}}{\left(k^{2}+k_{\perp}^{2} a^{2}\right)\left(k^{2}+f\right)}-\sigma \frac{\left(k^{2}+k_{\perp}^{2} a^{2}\right)^{2}}{k^{2}+f}-\frac{g \delta}{k^{2}+f}+\frac{i \delta k}{k^{2}+f}-\frac{2 i g k}{\left(k^{2}+k_{\perp}^{2} a^{2}\right)\left(k^{2}+f\right)}\right)=0
\end{aligned}
$$

where $\sigma=\frac{1}{\left(1+\zeta_{e} Z\left(\zeta_{e}\right)\right)} \frac{k_{\| 0}^{2}}{F_{0}^{\prime} a} \frac{\omega}{\omega_{* e}^{\prime} a} \frac{\rho_{s}^{2}}{a^{2}}$. In order for the inverse Fourier transform to exist, $\hat{\Phi}$ in Eq. (16) must satisfy the boundary conditions $\lim _{k \rightarrow \pm \infty} \hat{\Phi}(k) / k=0$.

Note that $\sigma, \delta, f$, and $g$ in Eq. (16) are complex. After the following transformation of variables, $\hat{\Phi} \equiv \Psi \frac{\left(k^{2}+k_{\perp}^{2} a^{2}\right)}{\left(k^{2}+f\right)^{3 / 4}} \exp \left(-i \frac{\delta k}{2}-i \frac{g}{2 f^{1 / 2}} \tan ^{-1}\left(\frac{k}{f^{1 / 2}}\right)\right)$, Eq. (16) acquires the form of Schrödinger's equation,

$$
\frac{d^{2} \Psi}{d y^{2}}+(\epsilon-U(y)) \Psi=0
$$

with the normalized independent variable $y \equiv k / k_{\perp} a$, energy $\epsilon=\tilde{\delta}^{2} / 4, \tilde{\delta}=\delta k_{\perp} a$, and the potential

$$
\begin{aligned}
U(y)= & \tilde{\sigma} \frac{\left(y^{2}+1\right)^{2}}{y^{2}+\tilde{f}}-\frac{-\frac{3}{4} y^{6}+y^{4}\left(-\frac{7}{2} \tilde{f}+\frac{5}{2}\right)+y^{2}\left(\frac{1}{4}+2 \tilde{f}-2 \tilde{f}^{2}\right)+\tilde{f}^{2}-\frac{\tilde{f}}{2}}{\left(y^{2}+1\right)^{2}\left(y^{2}+\tilde{f}\right)^{2}}-\frac{\tilde{g}^{2}}{4\left(y^{2}+\tilde{f}\right)^{2}}+\frac{\tilde{g} \tilde{\delta}}{2\left(y^{2}+\tilde{f}\right)}, \\
& -\frac{i \tilde{\delta} y}{2} \frac{\left(y^{2}+2 \tilde{f}-1\right)}{\left(y^{2}+1\right)\left(y^{2}+\tilde{f}\right)}+\frac{i \tilde{g} y}{2} \frac{3 y^{2}+6 \tilde{f}+1}{\left(y^{2}+1\right)\left(y^{2}+\tilde{f}\right)^{2}}
\end{aligned}
$$

with $\tilde{\sigma}=\sigma k_{\perp}^{4} a^{4}, \tilde{f}=f /\left(k_{\perp}^{2} a^{2}\right)$, and $\tilde{g}=g /\left(k_{\perp} a\right)$. The first four terms in Eq. (18) are even and the next two are odd with respect to $y$. Even when $m=1$, all terms remain regular as $|y| \rightarrow 0$ because $\tilde{f}$ is finite there due to the radial profiles of $\iota, n$, and $G$ (see Appendix A). For the small values of $y$ the form of the potential is defined by the second term in Eq. (18), which basically describes a potential well centered at $y=0$ with the minimum equal to $U_{\text {min }}=-1+1 /(2 \tilde{f})-\frac{\tilde{g}^{2}}{4 \tilde{f}^{2}}+\frac{\tilde{g} \tilde{\delta}}{2 \tilde{f}}$. Conversely, for large values of $y$ the first term in Eq. (18) dominates and asymptotically describes the harmonic oscillator potential. Consequently, solutions of Eq. (17) have discrete spectrum, which for large values of $\epsilon$ is

$$
\epsilon_{l} \approx \tilde{\sigma}^{1 / 2}(2 l+1)
$$

In practice, owing to the fact that the potential well for small values of $y$ described by the second, the third, and the fourth terms in Eq. (18) is too shallow to contain a bounded state (this is a manifestation of the absence of discrete modes in the monotonous parts of the Alfvén continuum, i.e. far from its maxima and minima, in the framework of ideal MHD with the parallel electric field set to zero), bounded states with small energies are also caused mainly by the first term in Eq. (18), so that Eq. (19) is generally applicable. The largest 
error in using Eq. (19) is for the fundamental solution with $l=0$, which can be improved by use of a variational method (see Appendix B).

Substituting the definition of $\tilde{\delta}$ and $\tilde{\sigma}$ in Eq. (19) one obtains the dispersion relation that determines frequency of the mode with toroidal and poloidal numbers $n$ and $m$, respectively, in terms of its radial number $l$. This dispersion relation can be written as

$$
h(\tilde{\omega})=(2 l+1) c
$$

where $\tilde{\omega} \equiv \omega / \omega_{0}, h(\tilde{\omega})=\frac{(1-\tilde{\omega})^{2}\left(\tilde{\omega}^{2} \Delta_{1}+\tilde{\omega}+1-\Delta_{1}+\Delta_{1} \Delta_{2}\right)^{2}}{4\left(\tilde{\omega}^{2}-1+\Delta_{2}\right)^{3 / 2} \tilde{\omega}^{1 / 2}}$, and $c=\left(\Delta_{1} \frac{\rho_{s 0}^{2}}{a^{2}} \frac{\left(\chi_{n} a\right)^{2}}{\left(1+\zeta_{e} Z\left(\zeta_{e}\right)\right)}\right)^{1 / 2}, \Delta_{1}=$ $-\left(\omega_{0} \chi_{n}\right) / \omega_{* e 0}^{\prime}, \Delta_{2}=\left(k_{\|}^{2} \overline{v_{A}^{2}}\right)_{0}^{\prime} /\left(\chi_{n} \omega_{0}^{2}\right)$, where $\chi_{n} \equiv-(d n / d r) / n$, are constants. In optimized stellarators with low shear $\Delta_{2}$ is close to unity (in shear-less case $\left(k_{\|}^{2} v_{A}^{2}\right)_{0}^{\prime} /\left(\chi_{n} \omega_{0}^{2}\right)=1$ exactly). However, since in such stellarators $k_{\|}$is also small due to the closeness of the $\iota$ to the magnetic field helicity, we keep $\Delta_{2} \neq 1$.

When the background electrons are close to the parallel resonance with the mode at $r_{0}$, i.e. $\omega \approx k_{\|} \sqrt{2 T_{e} / m_{e}}$, the mode growth rate can become relatively strong, $\gamma / \omega_{r} \simeq \mathcal{O}(1)$, where we used notation $\tilde{\omega} \equiv \omega_{r}+i \gamma$. When this is not the case and $\gamma / \omega_{r} \simeq \mathcal{O}\left(10^{-1}\right)$ or less, one can estimate the maximum growth rate that the family of the drift-kinetic Alfvén modes has. Indeed, in this case one can solve Eq. (20) perturbatively, first finding the real part of the frequency, calculating then the growth rate in terms of the real frequency and finding its maximum over the range of possible values of $\omega_{r}$. From Eq. (20) it follows that this procedure leads to

$$
\gamma_{\max }=\max _{\omega_{r}} \mathcal{F}\left(\omega_{r}\right)
$$

where

$$
\begin{aligned}
\mathcal{F}\left(\omega_{r}\right) & =\frac{\operatorname{Im}(c)}{\operatorname{Re}(c)} \frac{h\left(\omega_{r}\right)}{\partial h\left(\omega_{r}\right) / \partial \omega_{r}} \\
& =\frac{2 \omega_{r}\left(\omega_{r}-1\right)\left(\omega_{r}^{2} \Delta_{1}+\omega_{r}+1-\Delta_{1}+\Delta_{1} \Delta_{2}\right)\left(\omega_{r}^{2}+\Delta_{2}-1\right)}{5 \omega_{r}^{5} \Delta_{1}+\omega_{r}^{4}\left(1-\Delta_{1}\right)+8 \omega_{r}^{3} \Delta_{1}\left(\Delta_{2}-1\right)+7 \omega_{r}^{2} \Delta_{2}+3 \omega_{r} \Delta_{1}\left(\Delta_{2}-1\right)^{2}+\left(\Delta_{2}-1\right)\left(1-\Delta_{1}+\Delta_{1} \Delta_{2}\right)}
\end{aligned} .
$$

\section{NUMERICAL RESULTS}

In order to verify the outcomes of the analytical analysis we investigate Eqs. (12) and (14) by a Ritz-Galerkin method with B-splines as finite element basis. One seeks solutions of these equations in the form of an expansion in terms of a B-spline basis, $\Phi(r)=\sum_{n=0}^{N} a_{n} B_{n}(r)$ and $\Psi(r)=\sum_{n=0}^{N} b_{n} B_{n}(r)$, where $a_{n}$ and $b_{n}$ are coefficients of the expansion and $B_{n}$ are 
splines of desired order (in this particular code we used splines of the fourth order). By substituting $\Phi$ and $\Psi$ in this form in Eqs. (12) and (14) and by applying a projection operator on basis element $B_{m}(r)$ one obtains a system of linear equations for the complex coefficients of the form $\mathbf{M}(\omega) \cdot(\mathbf{a}, \mathbf{b})^{T}=0$, where $\mathbf{M}$ is a $2 N \times 2 N$ matrix which depends on the frequency. Eqs. (12) and (14) are written self-adjoint form, i.e. $\left(B_{m}, \hat{L} B_{l}\right)=\left(B_{l}, \hat{L} B_{m}\right)$, where $(f(r), g(r))=\int_{0}^{a} f(r) g(r) r d r$, and $\hat{L}$ denotes an appropriate linear operator in the equations. This form is particularly suitable for the numerical solving of the equations. Roots of the characteristic equation $\operatorname{det}[\mathbf{M}(\omega)]=0$ correspond to the eigenfrequencies of the system, and solving for the coefficients for each eigenfrequency by either inverse iteration or single value decomposition algorithms provides the radial structure of eigenfunction. It is worth noting that a direct calculation of the determinant in the characteristic equation is numerically inefficient and error prone. It is much more robust to use the fact that the determinant of a matrix equals the product of all its eigenvalues. Then, by monitoring the smallest eigenvalue of the matrix $\mathbf{M}(\omega)$ and finding values of $\omega$ where it vanishes, one is able to find the roots of the characteristic equation $[14,16]$.

As an example we use a plasma with parameters and profiles relevant to HSX, which is an operating optimized stellarator with low shear (Ref. [5]). Recent experiments on this facility demonstrated an unstable mode with frequency in the lowest part of the Alfvénic spectrum in the presence of a heated electron component of the background plasma (e.g., Ref. [3]), which makes this device an interesting system in context of the present study. The background magnetic field strength is chosen to be equal to $\bar{B}=0.5 \mathrm{~T}$, and the rotational transform $\iota=\iota_{0}+\left(\iota_{1}-\iota_{0}\right)(r / a)^{2}$ with $\iota_{0}=1.05$ and $\iota_{1}=1.1$. The appropriate mode has then $n=1$ and $m=1$. The particle density of the background plasma is taken to be $n(r)=n_{0}+\left(n_{1}-n_{0}\right)\left(\tanh \left[\left(r / a-s_{*}\right) / \Delta\right]-\tanh \left[-s_{*} / \Delta\right]\right) /\left(\tanh \left[\left(1-s_{*}\right) / \Delta\right]-\tanh \left[-s_{*} / \Delta\right]\right)$ with $n_{0}=2 \times 10^{18} m^{-3}, n_{1}=0.01 n_{0}, s_{*}=0.3$, and $\Delta=0.4$, the background temperature chosen is $T_{e}(r)=$ const $=1.5 \mathrm{keV}$, and $T_{i}(r)=$ const $=20 \mathrm{eV}$ (so that our assumption about cold ions is well satisfied). The background ion species were assumed to be deuterium. The corresponding profiles for the Alfvén continuum, the electron diamagnetic frequency, and $k_{\|} v_{t h e}$, where $v_{t h e}=\sqrt{2 T_{e} / m_{e}}$, are shown in Fig. 1 . The basic parameters in Eq. (20) calculated from these profiles are $r_{0} / a=0.64, \Delta_{1}=1.6, \Delta_{2}=1.6$, and $c=0.28-0.12 i$.

As mentioned in Section III, the local analysis developed here assumes that $\omega^{2}>$ $\left(k_{\|}^{2} \overline{v_{A}^{2}}\right)_{\min }$, it turns out that there also do not seem to be numerical solutions for which 
this condition fails. For the case in question there are three radial eigenfunctions which satisfy it (the corresponding radial eigenfunctions are plotted in Figs. 2 and 3). Note that the eigenfunctions are quite broad in the radial domain and therefore have global appearance. These solutions exhibit good agreement with Eq. (20) (see Figs. 4 and 5). As predicted, the largest error is found for the fundamental state with $l=0$. Although the real part of the ratio between the numerically calculated energy of this state is not so far from the prediction of Eq. (20), the complex phase of this ratio is comparable with the complex phase of the energy itself (see Fig. 5). This discrepancy is caused by the fact that Eq. (20) accounts only for the "harmonic oscillator" part of the total potential, while the fundamental state is also influenced by the potential well described by the second and third terms in Eq. (18). However, since this potential well is not able to contain a bounded state by itself as it was mentioned above (the appropriate parameters of the potential being in this case $\tilde{g} \approx-0.38$, $\tilde{f} \approx 0.7$, and $\tilde{\delta} \approx 0.41$ ), this state ultimately arises also due to the "harmonic oscillator" part of the potential. The real frequency and the growth rate calculated from Eq. (20) using the MATHEMATICA software package (Ref. [18]) still yield relatively good agreement with numerical predictions (see Figs. 6 and 7). Whereas the prediction for the energy of the fundamental state can be improved by using the technique described in Appendix B, this solution is not the most important one as it has relatively small growth rate. The other solutions with $l=1$ and $l=2$ provide much better agreement with Eq. (20) (see Figs. 4 and 5), the $l=1$ eigenfunction being in particularly good accord with the predicted value of energy and the corresponding values for the real part of the frequency along with the growth rate (see Figs. 6 and 7). The latter numerical solution has the largest growth rate. The numerical solution with $l=2$ seems to depart from the prediction of the analysis, which is apparently due to the limitations of the local analysis and non-local corrections not considered in the local approximations of the analysis. Indeed, from the definition of $x$ after Eq. (15) one can see that it expresses the distance from the point where the function $F$ describing the Alfvén continuum vanishes. The linear expansion of the coefficients in Eq. (12) adopted in the local analysis is no longer valid when the quadratic terms start to become comparable with the linear ones, which occurs for $\left(r-r_{0}\right) / a \approx(d n / d r)\left(r=r_{0}\right) / a\left(d^{2} n / d r^{2}\right)\left(r=r_{0}\right) \approx 0.35$. Since the $l=2$ solution has $\operatorname{Re}(\omega) / \omega_{0} \approx 0.41$ and $\omega_{A m i n} / \omega_{0} \approx 0.35$, it follows from Fig. 1 that the distance between the point where the Alfvén continuum vanishes, $r_{*} / a$, to $r_{0} / a$ is $\left(r_{0}-r_{*}\right) / a \approx 0.4$, so that the linear expansion breaks down. On the other hand, the distance 
between $r_{*} / a$ to the point $r / a=0$, where global boundary condition clearly overrides the local assumptions, is just $r_{*} / a \approx 0.2$. The width of the eigenfunction from the local analysis can be approximated as $\Delta x \approx \sigma^{1 / 4} \approx 0.16$. Consequently, the assumption of the local analysis that $x \rightarrow-\infty$ marginally holds for this solution and the global boundary condition at the axis starts to heavily influence the solution. Given these limitations of the local analysis, its agreement with the numerical prediction is surprisingly good. Further, by assuming validity of the local analysis for the modes with $l>2$ one can see that the predicted growth rate is also peaked with respect to the radial number of a mode with the maximum growth rate close to the numerical value, but reached for $l=4$ rather than $l=1$ deduced from the numerical solutions (see Fig. 7 (left)). Finally, applying the approximated formula given in Eqs. (21) and (22) with the corresponding $\mathcal{F}$ function plotted for the chosen parameters in Fig. 7 (right), one can see that the estimate for the growth rate given by that formula also

yields a value $\gamma_{\max } \approx 1.02 \times 10^{-1}$ close to the numerical result. Although direct comparison with the experiment is not possible due to the limitations of the analysis conducted in this paper (e.g., distribution function of the background electron component during the ECRH heating in HSX differs significantly from the Maxwellian), this study demonstrates that there do exist unstable modes in the frequency range of interest which are due to the kinetic rather than the MHD effects, notwithstanding their global appearance in the radial domain.

\section{CONCLUSIONS}

In this paper a mode in the lowest part of the Alfvén spectrum possessing helicity close to the background magnetic field rotational transform in a plasma with low shear has been considered. It turns out that the frequency of such a shear Alfvén mode can be so low that it can couple by means of the parallel electric field, usually neglected for MHD modes, with the drift mode in an inhomogeneous plasma. A local analysis for a case of coupling between the modes with same mode numbers has been developed and the sideband response is demonstrated to be insignificant for the investigated mode. The analysis predicts the existence of unstable drift-kinetic Alfvén modes with potentially large growth rates propagating in the direction of electron diamagnetic drift when the local electron diamagnetic frequency curve crosses the Alfvén continuum from above at a value larger than the minimum of the 
latter. A numerical solution of the equations governing the shear Alfvén dynamics in the presence of a finite parallel electric field was investigated for a set of parameters relevant to the optimized stellarator HSX, where electromagnetic activity in the lowest part of the Alfvénic spectrum was observed experimentally. A good agreement between the numerical solutions and the predicted values for the real part of the frequency and the growth rate was demonstrated. Although the analytical analysis differs from the numerical solution in its prediction of the exact radial number for the function having the maximum growth rate (presumably, due to the limitations of the local analysis), it yields correct qualitative behavior for the dependence of the peaked growth rate, and the peak value for the growth rate agrees well with the numerical prediction. The modes described here can appear to have global radial character and thus can possibly be confused with some other MHD modes in the same frequency range (e.g., Ref. [17]) when interpreting experimental data.

\section{Acknowledgment}

The author gratefully acknowledges P. Helander, A. Könies, and J. Nührenberg for useful discussions and comments on the manuscript. 
Let us define $F(r)=\omega^{2} / \overline{v_{A}^{2}}(r)-k_{\|}^{2}(r)$. Then, the left hand side of Eq. (12) can be written as

$$
F \frac{\partial^{2} \Phi}{\partial r^{2}}+\left(F^{\prime}+\frac{F}{r}\right) \frac{\partial \Phi}{\partial r}-\left(\frac{m^{2} F}{r^{2}}+\frac{F^{\prime}}{r}-G\right) \Phi .
$$

To obtain the coefficients $f$ and $g$ in Eq. (15) we expand the bracketed factor in the third term of this expression around the $r=r_{0}$, and take the dominant terms under the assumption that $F_{0}$ is small. We note that

$$
g_{0} \equiv\left(\frac{m^{2}}{r^{2}} F+\frac{F^{\prime}}{r}-G\right)\left(r=r_{0}\right) \approx \frac{F_{0}^{\prime}}{r_{0}}-G_{0}=\frac{m k_{\| 0}}{r_{0} R_{0}}\left(\iota_{0}^{\prime}+r_{0} \iota_{0}^{\prime \prime}\right) .
$$

Similarly,

$$
\begin{aligned}
\left(\frac{m^{2}}{r^{2}} F+\frac{F^{\prime}}{r}-G\right)^{\prime}\left(r=r_{0}\right) \approx & \frac{\left(m^{2}-1\right) F_{0}^{\prime}}{r_{0}}+\frac{F_{0}^{\prime \prime}}{r_{0}}-G_{0}^{\prime} \\
\equiv & \frac{\left(m^{2}-1\right)}{r_{0}^{2}}\left(\omega^{2}\left(\frac{1}{v_{A}^{2}}\right)_{0}^{\prime}-\left(k_{\|}^{2}\right)_{0}^{\prime}\right)+g_{1} \\
= & \frac{\left(m^{2}-1\right)}{r_{0}^{2}}\left(\omega^{2}\left(\frac{1}{\overline{v_{A}^{2}}}\right)_{0}^{\prime}-\left(k_{\|}^{2}\right)_{0}^{\prime}\right)+\frac{\omega^{2}}{r_{0}^{2}}\left(\frac{1}{\overline{v_{A}^{2}}}\right)_{0}^{\prime} \\
& +\frac{m k_{\| 0}}{r_{0} R_{0}}\left(-3 \frac{\iota_{0}^{\prime}}{r_{0}}+\iota_{0}^{\prime \prime}+r_{0} \iota_{0}^{\prime \prime \prime}\right)+\frac{m^{2}}{r_{0} R_{0}^{2}}\left(\left(\iota_{0}^{\prime}\right)^{2}+r_{0} \iota_{0}^{\prime} \iota_{0}^{\prime \prime}\right)
\end{aligned}
$$

Thus, using the definitions of Eq. (15), it is straightforward to see that $f=\frac{\left(m^{2}-1\right) a^{2}}{r_{0}^{2}}+\frac{a^{2} g_{1}}{F_{0}^{\prime}}$ and $g=-\frac{a}{F_{0}^{\prime}} g_{0}$ with $g_{0}$ and $g_{1}$ defined by Eqs. (A2) and (A3). 


\section{Appendix B: Estimate of the energy value for the fundamental solution}

Proceeding in the spirit of Ref. [13], one starts with an observation that the condition of zero variation of the functional $F=\int_{-\infty}^{\infty} d y S\left(\Psi, \Psi_{y}\right)$, where $S \equiv \Psi_{y}^{2}-(\epsilon-U(y)) \Psi^{2}$, is fulfilled through the Euler-Lagrange equations for such functions $\Psi$, which satisfy the Schrödinger's equation, Eq. (17). It follows also that for such functions $\Psi$ the functional $F$ vanishes. Therefore, by taking $\Psi_{\alpha}=\exp \left(-\alpha y^{2} / 2\right)$ as a trial function for the fundamental state and solving the appropriate equations

$$
\left\{\begin{array}{l}
F=0 \\
\partial F / \partial \alpha=0
\end{array}\right.
$$

one can obtain a good estimate for energy of the fundamental state $\epsilon_{0}$. Let us illustrate how this approach works for a case with $m \gg 1$. Taking $\tilde{f}$ in Eq. (18) as $\tilde{f} \approx 1 \gg \tilde{g}$, and thus neglecting the terms proportional to $\tilde{g}, U$ in Eq. (18) becomes

$$
U(y)=\tilde{\sigma}\left(1+y^{2}\right)+\frac{3 y^{2}-2}{4\left(1+y^{2}\right)^{2}} .
$$

Eqs. (B1) yield

$$
\left\{\begin{array}{c}
\frac{\alpha^{1 / 2}}{2}-\frac{\epsilon_{0}}{\alpha^{1 / 2}}+\frac{\tilde{\sigma}}{2 \alpha^{3 / 2}}(2 \alpha+1)-I(\alpha)=0 \\
\frac{1}{4 \alpha^{1 / 2}}+\frac{\epsilon_{0}}{2 \alpha^{3 / 2}}-\frac{\tilde{\sigma}}{4 \alpha^{5 / 2}}(2 \alpha+3)-\frac{\partial I}{\partial \alpha}=0
\end{array}\right.
$$

where $I(\alpha)=\frac{1}{\sqrt{\pi}} \int_{-\infty}^{\infty} d y e^{-\alpha y^{2}} \frac{-3 y^{2}+1}{4\left(y^{2}+1\right)^{2}}=\frac{5}{4} \alpha^{1 / 2}-\frac{\sqrt{\pi}}{8}(1+10 \alpha) \operatorname{erfc}\left(\alpha^{1 / 2}\right)$. Solving Eqs. (B3) for $\epsilon_{0}$, one obtains the fundamental energy level. When $|\tilde{\sigma}| \ll 1$, one can use the small argument expansion for $I(\alpha)$, so that solution of Eqs. (B3) results in $\epsilon_{0} \approx \frac{3}{2}\left(\frac{\sqrt{\pi}}{8}\right)^{2 / 3} \tilde{\sigma}^{1 / 3}+\left(\frac{8}{\sqrt{\pi}}\right)^{2 / 3} \tilde{\sigma}^{2 / 3}$. 
[1] C.-Y. Teo, A. Weller, C. Konrad, W7-AS Team, NBI Team, Nuclear Fusion, 38(3), 409 (1998)

[2] A. Weller, M. Anton, J. Geiger, M. Hirsch, R. Jaenicke, A. Werner, C. Nührenberg, E. Sallander, and D.A. Spong, Physics of Plasmas, 8(3), 931 (2001)

[3] D.L. Brower, C. Deng, D.A. Spong, A. Abdou, A.F. Almagri, D.T. Anderson, F.S.B. Anderson, W. Guttenfelder, K. Likin, S. Oh, V. Sakaguchi, J.N. Talmage, K. Zhai, Proc. of 32nd EPS Conference on Plasma Phys. Tarragona, ECA Vol.29C, O-2.001 (2005)

[4] A.B. Mikhailovskii and L.I. Rudakov, Sov. Phys. JETP 17, 621 (1963)

[5] F.S.B. Anderson, D.T. Anderson, A. Almagri, P.G. Matthews, J.N. Talmage, and J.L. Shohet, Fusion Technol. 27, 273 (1995)

[6] A.H. Boozer, Phys. Fluids 24, 1999 (1981)

[7] D.A. Garren and A.H. Boozer, Phys. Fluids B 3, 2805 (1991)

[8] Ya.I. Kolesnichenko, V.V. Lutsenko, H. Wobig, Yu.V. Yakovenko, and O.P. Fesenyuk, Phys. Plasmas 8(2), 491 (2001)

[9] O.P. Fesenyuk, Ya.I. Kolesnichenko, V.V. Lutsenko, R.B. White, and Yu.V. Yakovenko, Phys. Plasmas 11, $5444(2004)$

[10] A.J. Brizard and T.S. Hahm, Rev. Mod. Physics 79,421 (2007)

[11] M.N. Rosenbluth and P.H. Rutherford, Phys. Rev. Lett. 34, 1428 (1975)

[12] Y.M Li, S.M. Mahajan, and D.W. Ross, Physics of Plasmas, 30(5), 1466 (1987)

[13] S.M. Mahajan, Physics of Fluids, 27(9), 2238 (1984)

[14] Ph. Lauber, S. Günter, and S.D. Pinches, Physics of Plasmas, 12, 122501, 1 (2005)

[15] B.D. Fried and S.D. Conte, The Plasma Dispersion Function (Academic Press, New York, 1961)

[16] W.M. Tang, G. Rewoldt, Physics of Fluids B, 5, 2451 (1993)

[17] Ya.I. Kolesnichenko, V.V. Lutsenko, A. Weller, A. Werner, Yu.A. Yakovenko, J. Geiger, and O.P. Fesenyuk, Phys. Plasmas 14, 102504-1 (2007)

[18] S. Wolfram, The Mathematica Book (Wolfram Media, 1996) 


\section{FIGURE CAPTIONS}

FIG. 1. Radial dependence of the local values for the essential frequencies in the system normalized to the global minimum of the shear Alfvén continuum for the mode with $m=1$ and $n=1$. Shear Alfvén continuum is plotted with the solid curve, electron diamagnetic drift frequency curve with the dashed curve, and $k_{\|} v_{\text {the }}$ with the dotted curve.

FIG. 2. Real part of the potential corresponding to the three different radial eigenfunctions of DKAE obtained in the numerical solution. The real part of the eigenfunctions is normalized to unity.

FIG. 3. Imaginary part of the potential of the same eigenfunctions as displayed in Fig. 3.

FIG. 4. Verification of Eq. (20) with the results obtained numerically. The squares connected with the solid line display the numerical values and the triangles connected with the dashed line display the analytically predicted values.

FIG. 5. Discrepancy of the Eq. (20) with the numerical predictions. Imaginary part of $h(\tilde{\omega}) / c$ in Eq. (20) calculated with the numerical values of the eigenfrequency has a relatively large imaginary part for the fundamental solution with no radial nodes indicating a significant digression from the prediction of Eq. (20). On the opposite, the other two solutions show very good agreement with Eq. (20). For comparison sine of the complex phase for the right hand side of Eq. (20) is plotted with the dashed line.

FIG. 6. Numerical values of the normalized real part of the frequency for the modes showed in Figs. 2 and 3 (squares connected with the solid line) plotted against the analytical predictions obtained with solving Eq. (20) (triangles connected with the dashed line).

FIG. 7. Left: numerical values of the normalized growth rate of the frequency for the modes showed in Figs. 2 and 3 (squares connected with the solid line) plotted against the analytical predictions obtained with solving Eq. (20) (triangles connected with the dashed line). The predicted values are extended to the radial numbers beyond 
the numerically existing to demonstrate that the predicted values are also peaked. Right: $\mathcal{F}$ function from Eq. (22) approximating dependence of the growth rate on the real part of the mode frequency calculated for the parameters of the example case. 


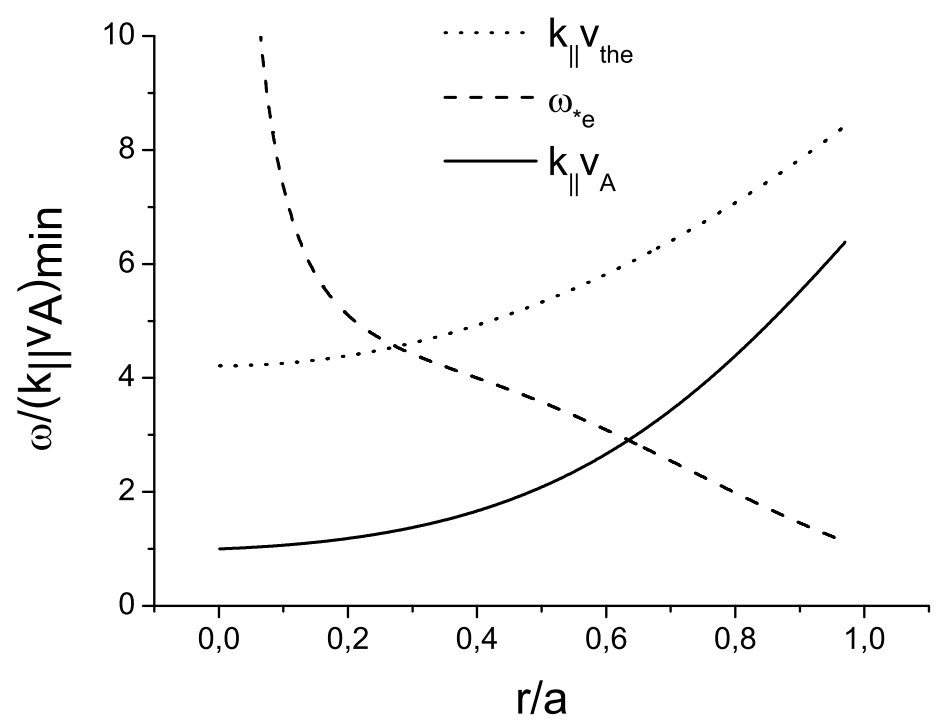

FIG. 1: 


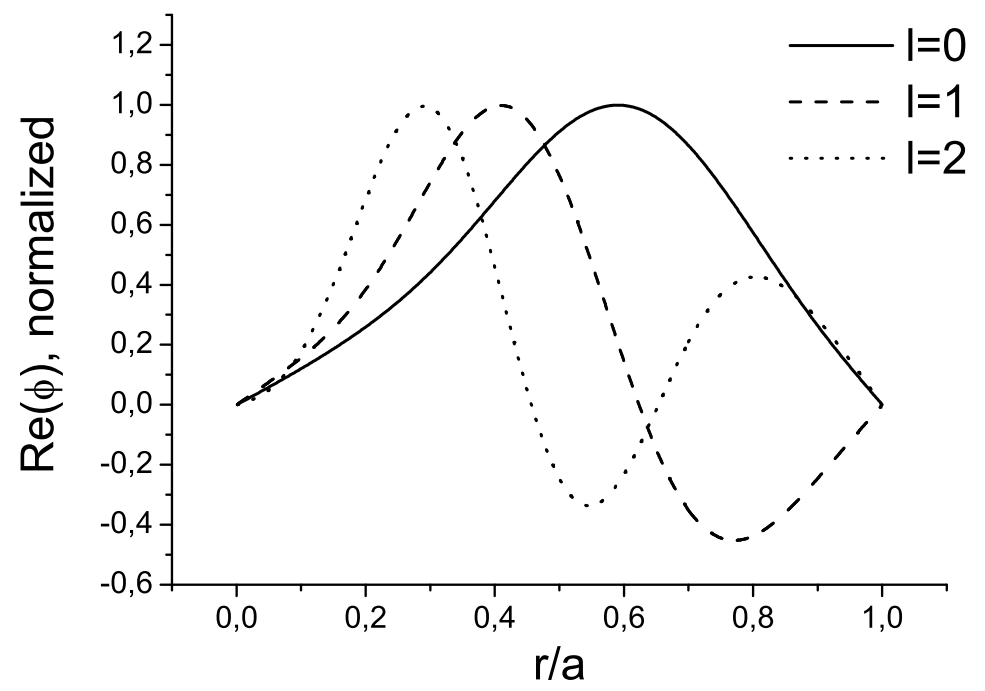

FIG. 2: 


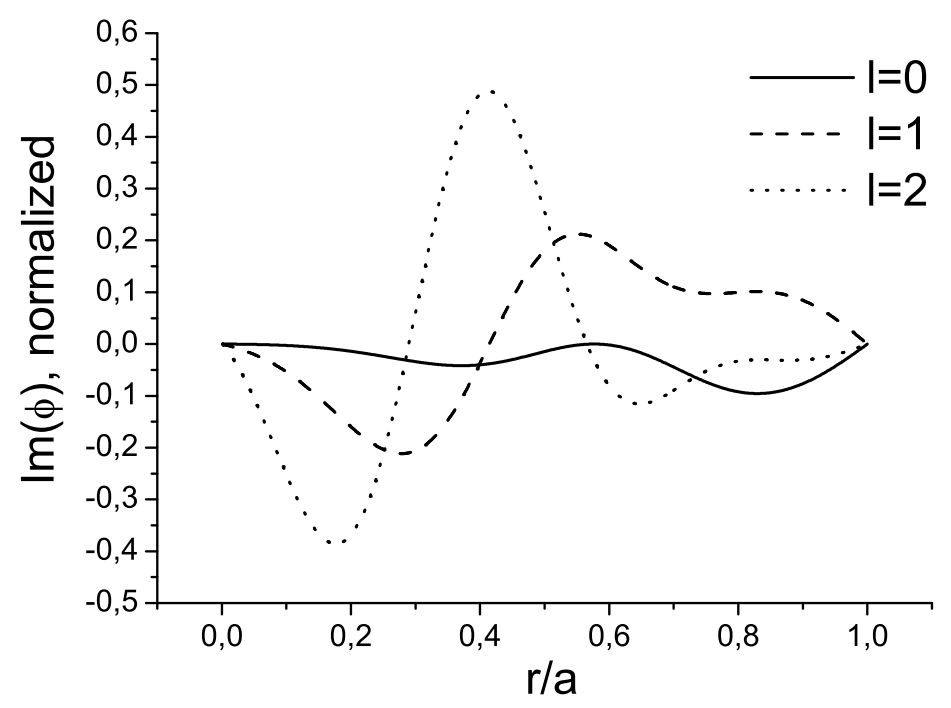

FIG. 3: 


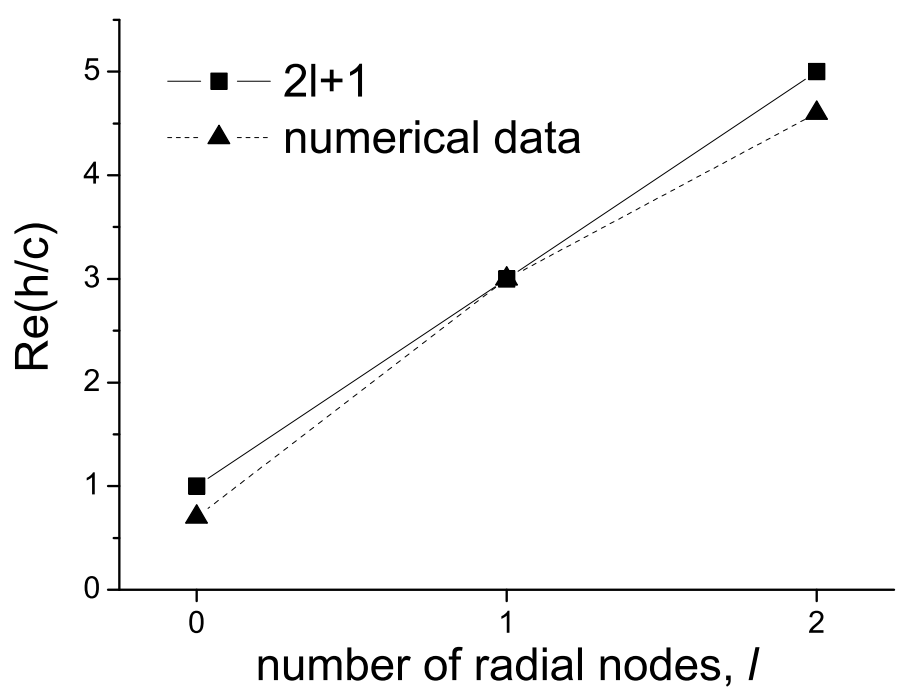

FIG. 4: 


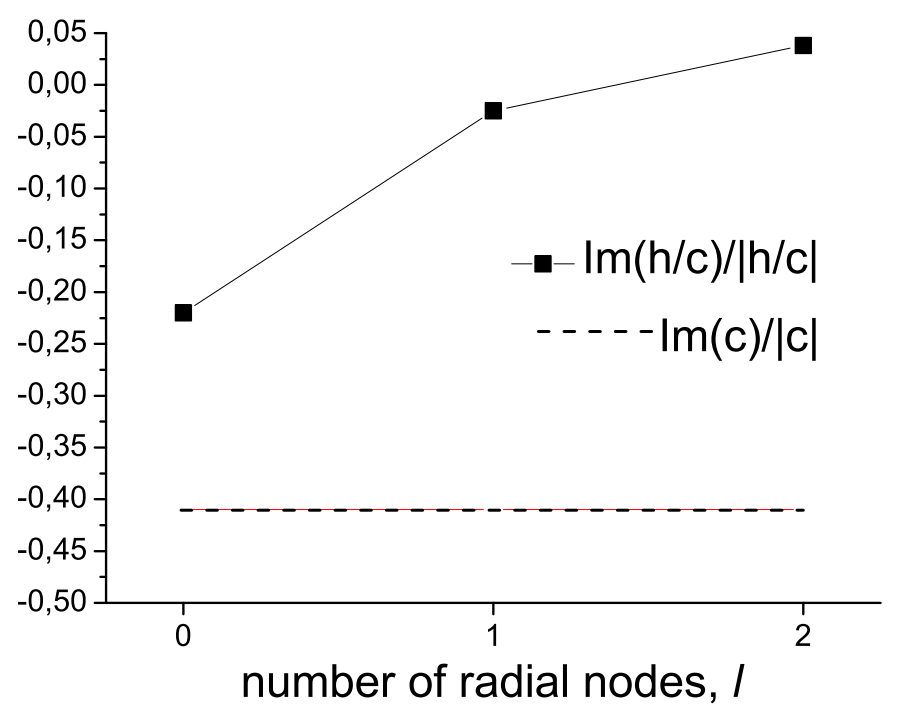

FIG. 5: 


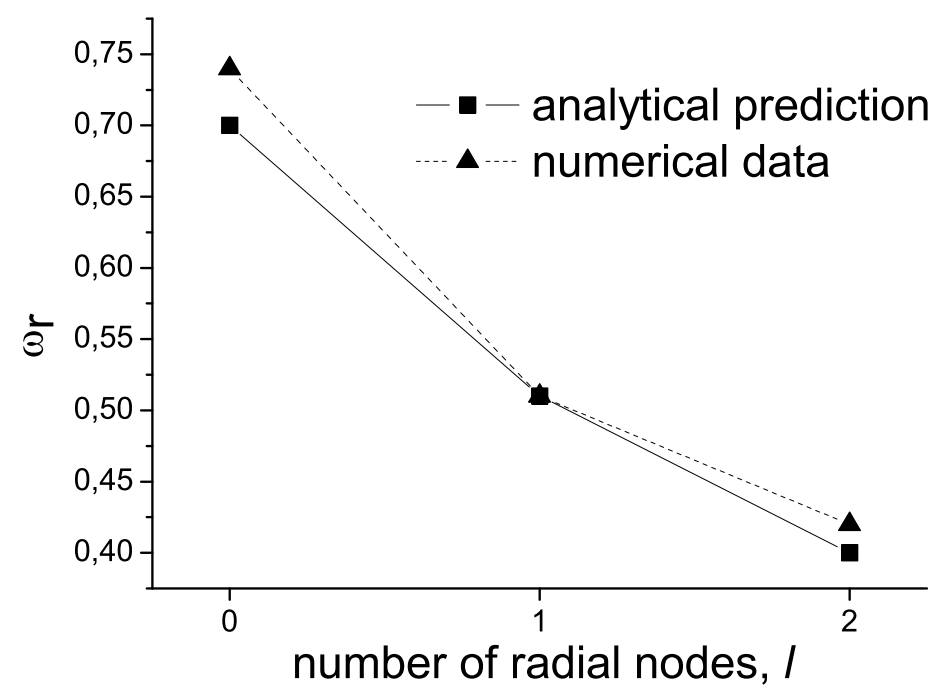

FIG. 6: 

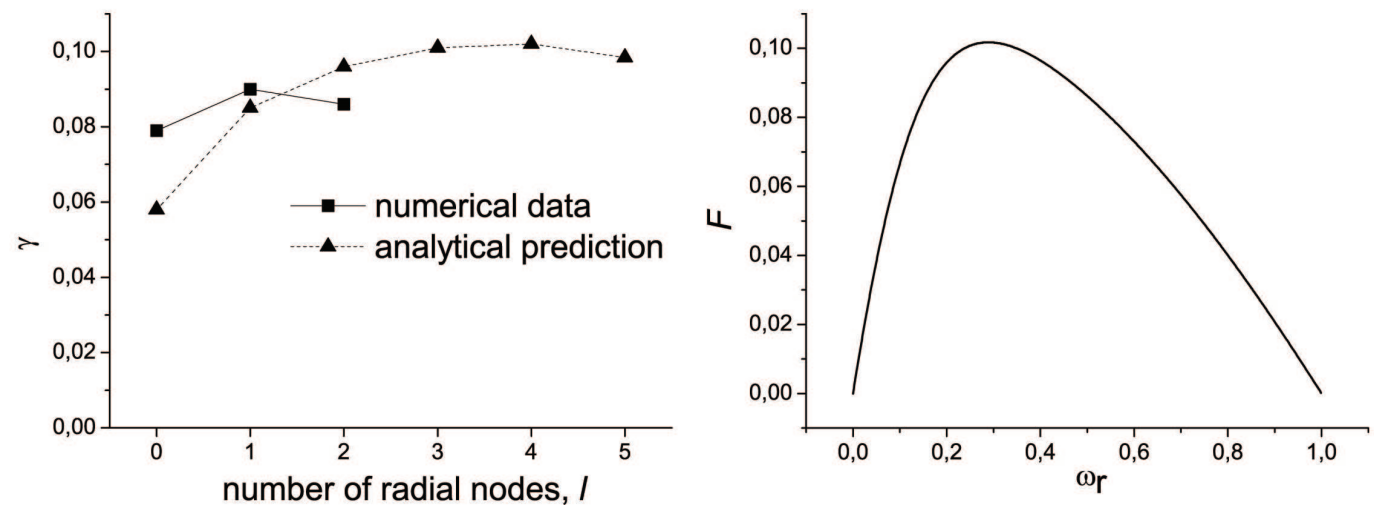

FIG. 7: 